\title{
Adalimumab in the treatment of non-infectious uveitis
}

\author{
Alicja Burek-Michalska,D, Anna Turno-Kręcicka ${ }^{2, E, F}$ \\ 1 University Hospital in Wrocław, Poland \\ ${ }^{2}$ Chair and Clinic of Ophthalmology, Wroclaw Medical University, Poland \\ A - research concept and design; $B$ - collection and/or assembly of data; $C$ - data analysis and interpretation; \\ $\mathrm{D}$ - writing the article; $\mathrm{E}$ - critical revision of the article; $\mathrm{F}$ - final approval of the article
}

\section{Address for correspondence}

Alicja Burek-Michalska

E-mail:alicja.burek@gmail.com

Funding sources

None declared

\section{Conflict of interest}

None declared

Received on March 4, 2020

Reviewed on March 24, 2020

Accepted on July 16, 2020

Published online on October 30, 2020

\begin{abstract}
Non-infectious uveitis (NIU) is a serious sight-threatening condition whose pathogenesis is often autoimmune in nature. It may manifest in any age group, though adults aged 20-50 are the group most often affected. It causes $5-10 \%$ of visual impairment worldwide. The epidemiology of some specific uveitis diseases varies worldwide, because they are influenced by genetic, environmental and socioeconomic factors. It can occur only in the eye or as a symptom of a systemic condition. The most common cause of NIU is HLA-B-27-associated anterior uveitis (4-32\%). The standard treatment for NIU is a local, topical and systemic steroid therapy in combination with immunomodulatory therapy. However, recently, a new drug - adalimumab, which is a tumor necrosis factor a (TNF-a) inhibitor - was approved by FDA in the treatment of NIU and is increasingly used to treat various conditions. Adalimumab has been proven in many studies to be safe and effective in the treatment of NIU associated with diverse systemic diseases.
\end{abstract}

Key words: non-infectious uveitis, adalimumab, biologic therapy, TNF-a inhibitors

Cite as

Burek-Michalska A, Turno-Kręcicka A. Adalimumab in the treatment of non-infectious uveitis. Adv Clin Exp Med. 2020;29(10):1231-1236. doi:10.17219/acem/125431

DOI

10.17219/acem/125431

\section{Copyright}

Copyright by Author(s)

This is an article distributed under the terms of the

Creative Commons Attribution 3.0 Unported (CC BY 3.0)

(https://creativecommons.org/licenses/by/3.0/) 


\section{Introduction}

Uveitis is a common cause of blindness worldwide, ${ }^{1}$ which in turn may be caused by infectious or non-infectious factors. ${ }^{2}$ Non-infectious uveitis (NIU) is often associated with an underlying systemic, vision-threatening disease. It is characterized by inflammation in the uveal tract and generally occurs more often in the developed world. The most common causes of NIU are HLA-B27associated anterior uveitis, sarcoidosis, Vogt-KoyanagiHarada syndrome, sympathetic ophthalmia, birdshot chorioretinopathy (BSCR), multifocal choroiditis, serpiginous choroiditis, and Behçet's disease (BD). ${ }^{2}$

Currently, the mainstay in the treatment of uveitis is steroid therapy, focused on suppressing the severity of inflammation. ${ }^{1,3}$ Although corticosteroids are often effective, ocular and/or systemic adverse effects limit their long-term use. ${ }^{4,5}$ Other therapeutic options for primary or secondary NIU include traditional immunosuppressants, such as cyclosporine (CsA), methotrexate (MTX), azathioprine (AZA), sulfasalazine (SSZ), and mycophenolate mofetil (MMF). However, a significant proportion of uveitis cases cannot be controlled only using corticosteroids and immunosuppressants. ${ }^{6}$ A new promising drug approved for the treatment of NIU in adults is adalimumab. ${ }^{5,7}$ Adalimumab is a recombinant human immunoglobulin monoclonal antibody that specifically binds to tumor necrosis factor $\alpha$ (TNF- $\alpha)^{1,6,8}$ Although there are other TNF- $\alpha$ inhibitors, such as etanercept and infliximab, adalimumab has been proven to have an advantage in therapeutic treatment, probably because of its higher affinity to bind with TNF- $\alpha$ than etanercept or infliximab. ${ }^{9}$

Tumor necrosis factors are a group of cytokines that are produced by activated macrophages, CD4+lymphocytes and natural killer cells. These cytokines are responsible for inducing inflammation and apoptosis and for inhibiting viral replication. ${ }^{10,11}$ Moreover, TNF- $\alpha$ is found to upregulate vascular endothelial growth factor (VEGF) production in choroidal endothelial cells, and VEGF is responsible for macular edema in uveitic patients. This explains the successful outcome of adalimumab treatment, reducing VEGF- $\alpha$ levels in the plasma by inhibiting TNF- $\alpha$ production in the treatment of uveitic macular edema. ${ }^{11,12}$ The pro-inflammatory cytokine TNF- $\alpha$ is thought to play a key role in uveitis inflammation, and aqueous humor and serum levels of TNF- $\alpha$ are upregulated in uveitis patients. ${ }^{6,13}$ Tumor necrosis factor $\alpha$ binds to specific membrane receptors (TNF- $\alpha$ receptors I and II). In the eye, TNF- $\alpha$ receptors I and II are located at the surface of the pigment epithelium cells of the iris, ciliary body and retina. ${ }^{11}$

\section{Adalimumab binds to TNF- $a$, inhibiting its pro-inflammatory and apoptotic action}

Apart from its beneficial effect, adalimumab may lead to severe side effects, the most frequent of which are infections. In a long-term analysis of 23,458 patients, there were reported cases of cellulitis, pneumonia, appendicitis, herpes zoster, urinary tract infection, gastrointestinal tract abscess, and gastroenteritis. Other severe side effects, such as tuberculosis, opportunistic infections, demyelinating disorders, lupus-like syndrome, and congestive heart failure, were rare. The incidence of malignancies among patients treated with adalimumab was similar to that of malignancies in a reference population. The overall rates of lymphoma were greater in a rheumatoid arthritis (RA) group treated with adalimumab, ${ }^{14}$ but RA increases the risk of lymphoma itself ${ }^{15}$ and the data showed that the incidence of lymphoma among RA patients treated with adalimumab was the same as among the RA patients who had never been treated with anti-TNF- $\alpha{ }^{16}$

\section{Clinical studies of adalimumab in non-infectious uveitis}

Ming et al. analyzed 3 randomized clinical trials and 20 non-randomized clinical trials in order to summarize the current evidence of the efficacy and safety of adalimumab in treating patients with NIU. ${ }^{3}$ The primary outcome of the analysis was a decrease in inflammatory activity. The inflammatory activity of uveitis was found to have decreased by $74 \%$ and $79 \%$ in their combined analysis of short and relatively long-term follow-up periods, respectively. In addition to this effectiveness, the meta-analysis revealed other promising results, demonstrating that adalimumab is a reasonable choice in the treatment of uveitis. It controlled the worsening of visual acuity $(88.8 \%$ of involved eyes), reduced the use of corticosteroids, significantly so in $3 / 4$ of the patients, and was generally well-tolerated, thus proving to be safe. Patients with juvenile idiopathic arthritis, BD and HLA-B-27-associated uveitis were studied.

A multicenter retrospective study was conducted by Bitossi et al. to assess the long-term ocular control of adalimumab in a population with non-infectious primary or secondary uveitis. ${ }^{6}$ The main goal of the study was to evaluate its steroid-sparing effect and co-treatment with diseasemodifying anti-rheumatism drugs (DMARDs). A total of 106 eyes were included. The effective ocular control was $83.7 \%$ and $83.3 \%$, after 6 and 12 months, respectively. Patients with poor baseline visual acuity remained stable or improved, while those with good vision acuity worsened. Other findings of the study were that the steroid dosages were reduced and that ocular control and the steroid-sparing effect were not influenced by the concomitant use 
of DMARDs. Mild or moderate side effects were reported in $6.6 \%$ of the patients.

The most significant studies on adalimumab in NIU are the VISUAL studies - multicenter randomized, controlled trials. ${ }^{4,17}$ The VISUAL I, VISUAL II and VISUAL III studies involved 217, 229 and 371 patients, respectively. In the first 2 rounds of the study, participants (aged $\geq 18$ years) with active, non-infectious intermediate or posterior uveitis or panuveitis were enrolled. The risk of treatment failure for participants in the adalimumab group was lower than that of the participants in the placebo group, by $44 \%$ in VISUAL I and by $48 \%$ in VISUAL II. The incidence of adverse effects in both studies was comparable between the placebo and adalimumab groups. The most common adverse effect was injection site reaction. There were also 2 cases of malignancies each in VISUAL I and VISUAL II; 2 participants had tuberculosis in VISUAL I and 3 in VISUAL II. One case of demyelinating disorder, lupus and lupus-like reaction was reported. However, the most relevant conclusion of these studies is that adalimumab lowered the risk of uveitic flare or visual acuity loss in the participants with active and controlled uveitis who were at risk of the long-term side effects of corticosteroids (CS).

The VISUAL III study lasted 78 weeks. As before, the main goal of the study was to assess the efficacy, safety and steroid- and immunomodulator-sparing effect of adalimumab. To determine its efficacy, anterior chamber activity, vitreous activity and mean best-corrected visual acuity (BCVA) were analyzed at 0, 12 and 78 weeks. The results revealed an improvement in ocular control inflammation and mean BCVA, but only in patients who had active uveitis initially. The BCVA remained stable over time in patients with inactive uveitis. For patients with active uveitis at study entry, the observed mean daily corticosteroid dose had decreased by $75 \%$ at week 78 and the mean dose of immunomodulators had decreased by $26 \%$ at week 78 in comparison with week 8 .

\section{Adalimumab in the treatment of selected disorders}

\section{HLA-B-27-related uveitis}

Most patients with HLA-B27-associated uveitis have an associated spondyloarthropathy (AS); the archetypal disease is ankylosing spondylitis. Other forms of spondyloarthropathy include reactive arthritis (Reiter's syndrome), arthritis associated with inflammatory bowel disease, psoriatic arthritis, undifferentiated spondyloarthropathy, and juvenile forms of these diseases. The most typical HLA-B27-related uveitis is a sudden onset, unilateral, recurrent, anterior uveitis. ${ }^{18}$ The pathogenic mechanism underlying HLA-B27 uveitis is strongly associated with pro-inflammatory cytokines, among which TNF- $\alpha$ plays a key role. Pérez-Guijo et al. proved that by analyzing samples of the aqueous humor concentration of TNF- $\alpha$ among HLA-B27-positive patients and B27-negative patients. The concentration of TNF- $\alpha$ in the aqueous humor of HLA-B27-positive patients was significantly higher than in those who were HLA-B27-negative. ${ }^{19}$

The most relevant study on anterior uveitis in active AS was an open-label, uncontrolled study on 1,250 patients. ${ }^{20}$ It was conducted to assess the effect of adalimumab on the frequency of anterior uveitis in active spondylitis. Adalimumab was administered every week for 12 weeks. Adalimumab effectiveness was assessed by measuring the rate of anterior uveitis flare. The effect was at least a $50 \%$ reduction in the anterior uveitis flare rate during adalimumab treatment in patients with active AS.

Kim et al. analyzed the medical records of 143 patients diagnosed with HLA-B27-positive AS who were taking an anti-TNF- $\alpha$ agent for at least 1 year. ${ }^{21}$ Among all the patients enrolled in this study, 45 were treated with adalimumab, while the others were administered infliximab and etanercept. Almost $3 / 4$ of the patients receiving adalimumab had a history of uveitis. The examination was done at baseline and after 1, 2 and 12 months of treatment. During the follow-up, uveitis inflammation decreased after $17.9 \pm 6$ days among all of the patients, as assessed according to the Standardization of Uveitis Nomenclature (SUN) Working Group. Almost $80 \%$ of the patients had no recurrence of uveitis during anti-TNF- $\alpha$ treatment. A decrease was observed in the number of systemic medications in $93.3 \%$ of the patients. Minor side effects were noted in 7 patients, while 1 case of tuberculosis was diagnosed. ${ }^{16}$

\section{Sarcoidosis}

Sarcoidosis is a multisystemic, idiopathic disorder that is characterized by the formation of immune granulomas in the involved organs. ${ }^{22}$ The eye is often the first organ affected by the disease, which can lead to severe visual impairment. Any part of the uvea can be involved in the pathogenic process, as well as other parts of the eye and its adnexa. Most sarcoid uveitis is bilateral and chronic. ${ }^{23}$

Erckens et al. enrolled 26 patients with sarcoidosis who were being treated with adalimumab due to refractory posterior uveitis. ${ }^{24}$ Before adalimumab was administered, all of the patients were treated with oral corticosteroids, and MTX as the second-line agent if there was no response to corticosteroids. The patients were examined at baseline and after 6 and 12 months of treatment. At baseline, they manifested various intraocular inflammatory signs, such as papillitis, macular edema, choroidal involvement, vasculitis, and vitritis. After 6 months, vitreous involvement and vasculitis resolved in all 26 patients; papillitis was resolved in 7 patients (of the 8 in whom it was present at baseline), while 1 had a partial response; macular edema resolved in 5 of 8 patients, while the remaining 3 had a partial response; choroidal involvement was initially present in 15 patients and resolved in 10 of them, 
while the remainder had a partial response. The final result was improvement in $85 \%$ of patients and stabilization in 15\%; there was no difference in the results after 6 or 12 months. Only 1 patient had a serious side effect at the injection site, which caused a subcutaneous mass to form.

In another open-label, multicenter study, 17 patients were enrolled to assess anti-TNF- $\alpha$ therapy among patients with uveitis related to sarcoidosis and refractory to standard treatment. The outcomes assessed after 2 years were visual acuity, macular thickness, intraocular inflammation, and a corticosteroid- and immunosuppressivesparing effect. All of them show improvement 2 years after the onset of adalimumab therapy. ${ }^{25}$

\section{Behçet's disease}

Behçet's disease is an idiopathic, chronic, multisystem inflammatory vasculitis characterized mainly by recurrent oral aphthous ulcers, genital ulcers and severe intraocular inflammation. ${ }^{26}$ Any part of the uvea can be involved in the pathogenic process, and the frequency of ocular involvement is in the range of 50-70\%. Clinical manifestations of acute inflammation are typically self-limiting over time with relapsing episodes of varying intensity. The frequency and duration of relapses are unpredictable and follow no discernible pattern of onset. Occasionally, the cardiovascular system, the central nervous system and the gastrointestinal tract can be involved in the pathogenic process. ${ }^{26}$ The strongest genetic factor related to $\mathrm{BD}$ is HLA-B-51 antigen. The presence of HLA-B-51 may lead to $\mathrm{BD}$ in both the adaptive and in nate immune responses. An increased expression of pro-inflammatory cytokines, including IL-1, IL-6, IL-8, and TNF- $\alpha$, among patients with $\mathrm{BD}$ has been reported, which proves the role of an innate immune response in the pathogenesis of $\mathrm{BD} .^{27}$

In a multicenter retrospective, observational study, the medical histories of 44 patients (66 eyes) with BDrelated uveitis were analyzed. ${ }^{28}$ The medical data of this study was analyzed at baseline, after 3 months of treatment and after 12 months. Almost all of the patients (97.5\%) had previously been treated with steroids; some of them (32.5\%) were treated with other biological agents, and some of them had taken DMARDs (77.5\%). The steroid treatment was continued with adalimumab in $92.5 \%$ of patients and with DMARDs in $42.5 \%$ of patients. Significant improvement was demonstrated in all of the factors under study, the number of flares, BCVA and optical coherence tomography (OCT) findings regarding central macular thickness and vasculitis, so visual, clinical, functional, and morphological improvement was observed. The other relevant finding of this study was that a combination of adalimumab and other immunosuppressive agents had no superior benefit over monotherapy. Patients co-administered with DMARDs had more flares, but this could also be due to a higher activity of the disease at baseline, making it necessary to administer the combination therapy early on.

\section{Birdshot chorioretinopathy}

Birdshot chorioretinopathy is a non-infectious, bilateral panuveitis. The pathogenic mechanism of BSCR is not well-understood; several pathways have been proposed, but there is definitely a strong association between the presence of the HLA-A29 molecule and BSCR. ${ }^{29}$ Almost all patients are Caucasian adults, and women are affected more often than men. ${ }^{30}$ The characteristic features of BSCR are yellow-white choroiditis spots. Most of the patients also have cystoid macular edema, caused by diffuse retinal vasculitis. ${ }^{30}$

Huis Het Veld et al. analyzed the medical records of 19 patients (38 eyes) with HLA-A29- positive BSCR who received adalimumab treatment. ${ }^{31}$ None of the patients enrolled in this study had responded to previous standard systemic immunomodulatory therapy. The analyzed records were from 1 year before adalimumab treatment, at baseline and after 1 year of treatment. The most relevant observation was an improvement in visual function after 1 year of adalimumab therapy. As in the previously mentioned studies, adalimumab was generally well-tolerated and allowed the dosages of co-administered immunosuppressants to be lowered. However, the ocular inflammation control was poorer than that of the other previously mentioned diseases. Only 2 of 9 patients experienced a complete remission of inflammation (as measured using OCT and fluorescein angiography).

In another retrospective case series study, the medical records of 3 patients were analyzed. ${ }^{32}$ All of the patients were HLA-A29-positive. Before adalimumab was introduced, all patients were treated with oral prednisolone (>10 mg) and 2 second-line immunosuppressive agents. The most relevant outcome was a significant decrease in central macular thickness after 6 and 12 months of adalimumab treatment. The BCVA improved in 4 eyes after the introduction of adalimumab, and improvement was noted in at least 1 electrodiagnostic parameter. The reduction in central macular thickness allowed the dosages of systemic immunosuppressive drugs to be lowered.

\section{Sympathetic ophthalmia}

Sympathetic ophthalmia is a rare, bilateral, non-necrotizing granulomatous uveitis. The incidence ranges from $0.2 \%$ to $0.5 \%$ following injury and $0.01 \%$ following intraocular surgery. ${ }^{33}$ The eye which has undergone trauma or surgery is the exciting eye, and the other eye is the sympathizing eye. The clinical manifestations may be anterior, cells and flare in the anterior chamber or mutton fat keratic precipitates, or posterior, vitritis with yellowish-white subretinal lesion, papillitis, choroiditis, exudative retinal detachment, or choroidal granulomas. A diffuse, granulomatous inflammatory response of the uveal tract is caused mainly by infiltration of the t-lymphocytes. ${ }^{34}$ So far, steroidotherapy alone or combined with other immunosuppressive agents, 
such as CsA or AZA, has been the mainstay of sympathetic ophthalmia treatment. ${ }^{33}$ However, Kim et al. and Hiyama et al. published some case reports proving that adalimumab can lead to successful outcomes in the treatment of sympathetic ophthalmia. ${ }^{35,36}$ So far, these are the only 2 papers concerning treatment with adalimumab in sympathetic ophthalmia to be published. Kim et al. published the case report of a young girl who developed sympathetic ophthalmia after an accidental injury to one eye. After initial treatment by local and systemic steroidotherapy and a minor decrease in inflammation activity, the girl developed weight gain and cushingoid habitus. The steroid treatment was tapered off and methotrexate was initiated. The patient continued to have inflammation, so adalimumab was administered. Within 3 months, the inflammation had completely resolved, and the steroids were discontinued. After 6 months of stability on adalimumab, methotrexate was tapered off and discontinued over the course of 6 months. After 18 months of adalimumab therapy, no evidence of recurrent inflammation was observed. ${ }^{35}$ Hiyama et al. reported 2 cases of sympathetic ophthalmia following trabeculectomy. ${ }^{36}$ In both cases, the initial treatment was steroids, but in the $1^{\text {st }}$ case, although the serous retinal detachment resolved, the steroids had to be discontinued because of diabetes mellitus. Methotrexate, the secondline treatment, was not tolerated, and while CsA was introduced, the serous retinal detachment relapsed. Lastly, adalimumab was administered, which led to a remission of serous retinal detachment and control of inflammation for 7 months. In the $2^{\text {nd }}$ case, serous retinal detachment did not resolve completely during steroid therapy, and steroids had to be discontinued because of the primary open-angle glaucoma. The second-line treatment, CsA was not tolerated, so adalimumab was introduced, leading to a remission of the serous retinal detachment and control of the ocular inflammation for 7 months. ${ }^{36}$

\section{Conclusions}

Based on the results of several clinical studies, adalimumab is a safe and promising therapy in NIU that preserves vision and controls ocular inflammation in the majority of associated systemic diseases. However, there are still some types of refractory uveitis, like BSCR, where complete remission of the inflammation is rarely achieved.

Moreover, adalimumab is also a reasonable choice as a steroid-sparing agent, limiting the ocular and systemic side effects that are associated with chronic corticosteroid use, as well as an immunosuppressant-sparing agent. In the majority of patients, it allows the disease to be controlled with minimal corticosteroid use. Even the side effects of adalimumab are rare and mild to moderate, though there is still a risk of severe side effects, such as infections and demyelinating disorders, which require cautious qualification for the treatment.

\section{ORCID iDs}

Alicja Burek-Michalska (D) https://orcid.org/0000-0001-9764-0757 Anna Turno-Kręcicka (D) https://orcid.org/0000-0001-6732-1851

\section{References}

1. Hasegawa E, Takeda A, Yawata N, Sonoda KH. The effectiveness of adalimumab treatment for non-infectious uveitis. Immunol Med. 2019;42(2):79-83. doi:10.1080/25785826.2019.1642080

2. Tsirouki T, Dastiridou A, Symeonidis C, et al. A focus on the epidemiology of uveitis. Ocul Immunol Inflamm. 2018;26(1):2-16. doi:10.1080 /09273948.2016.1196713

3. Ming S, Xie K, He H, Li Y, Lei B. Efficacy and safety of adalimumab in the treatment of non-infectious uveitis: A meta-analysis and systematic review. Drug Des Devel Ther. 2018;12:2005-2016. doi:10.2147/ DDDT.S160431

4. Goto H, Zako M, Namba K, et al. Adalimumab in active and inactive, non-infectious uveitis: Global results from the VISUAL I and VISUAL II trials. Ocul Immunol Inflamm. 2019;27(1):40-50. doi:10.1080/09273948. 2018.1491605

5. LaMattina KC, Goldstein DA. Adalimumab for the treatment of uveitis. Expert Rev Clin Immunol. 2017;13(3):181-188. doi:10.1080/1744666X. 2017.1288097

6. Bitossi A, Bettiol A, Silvestri E, et al. Adalimumab accounts for longterm control of noninfectious uveitis also in the absence of concomitant DMARD treatment: A multicenter retrospective study. Mediators Inflamm. 2019;2019:1623847. doi:10.1155/2019/1623847

7. Leal I, Rodrigues FB, Sousa DC, et al. Anti-TNF drugs for chronic uveitis in adults: A systematic review and meta-analysis of randomized controlled trials. Front Med (Lausanne). 2019;6:104. doi:10.3389/fmed. 2019.00104

8. Sheppard J, Joshi A, Betts KA, et al. Effect of adalimumab on visual functioning in patients with noninfectious intermediate uveitis, posterior uveitis, and panuveitis in the VISUAL-1 and VISUAL-2 trials. JAMA Ophthalmol. 2017;135(6):511-518. doi:10.1001/jamaophthalmol. 2017.0603

9. Hu S, Liang S, Guo H, et al. Comparison of the inhibition mechanisms of adalimumab and infliximab in treating tumor necrosis factor a-associated diseases from a molecular view. J Biol Chem. 2013; 288(38):27059-27067. doi:10.1074/jbc.M113.491530

10. Rifkin LM, Birnbaum AD, Goldstein DA. TNF inhibition for ophthalmic indications: Current status and outlook. BioDrugs. 2013;27(4):347-357. doi:10.1007/s40259-013-0022-9

11. Cordero-Coma M, Sobrin L. Antitumor necrosis factor-a therapy in uveitis. Surv Ophthalmol. 2015;60(6):575-589. doi:10.1016/j.survo phthal.2015.06.004

12. Goldhardt R, Rosen BS. Uveitic macular edema: Treatment update. Curr Ophthalmol Rep. 2016;4(1):30-37. doi:10.1007/s40135-016-0090-3

13. Jaffe GJ, Dick AD, Brézin AP, et al. Adalimumab in patients with active noninfectious uveitis. N Engl J Med. 2016;375(10):932-943. doi:10. 1056/NEJMoa1509852

14. Burmester GR, Panaccione R, Gordon KB, Mcllraith MJ, Lacerda APM. Adalimumab: Long-term safety in 23458 patients from global clinical trials in rheumatoid arthritis, juvenile idiopathic arthritis, ankylosing spondylitis, psoriatic arthritis, psoriasis and Crohn's disease. Ann Rheum Dis. 2013;72(4):517-524. doi:10.1136/annrheumdis-2011201244

15. Baecklund E, Iliadou A, Askling J, et al. Association of chronic inflammation, not its treatment, with increased lymphoma risk in rheumatoid arthritis. Arthritis Rheum. 2006;54(3):692-701. doi:10.1002/ art.21675

16. Hellgren K, Smedby KE, Feltelius N, Baecklund E, Askling J. Do rheumatoid arthritis and lymphoma share risk factors? A comparison of lymphoma and cancer risks before and after diagnosis of rheumatoid arthritis. Arthritis Rheum. 2010;62(5):1252-1258. doi:10.1002/ art.27402

17. Suhler EB, Adán A, Brézin AP, et al. Safety and efficacy of adalimumab in patients with noninfectious uveitis in an ongoing openlabel study: VISUAL III. Ophthalmology. 2018;125(7):1075-1087. doi:10. 1016/j.ophtha.2017.12.039

18. Martin TM, Rosenbaum JT. An update on the genetics of HLA-B27-associated acute anterior uveitis. Ocul Immunol Inflamm. 2011;19(2): 108-114. doi:10.3109/09273948.2011.559302 
19. Pérez-Guijo V, Santos-Lacomba M, Sánchez-Hernández M, CastroVillegas MDC, Gallardo-Galera JM, Collantes-Estévez E. Tumour necrosis factor-alpha levels in aqueous humour and serum from patients with uveitis: The involvement of HLA-B27. Curr Med Res Opin. 2004;20(2):155-157. doi:10.1185/030079903125002847

20. Rudwaleit M, Rødevand E, Holck P, et al. Adalimumab effectively reduces the rate of anterior uveitis flares in patients with active ankylosing spondylitis: Results of a prospective open-label study. Ann Rheum Dis. 2009;68(5):696-701. doi:10.1136/ard.2008.092585

21. Kim M, Won JY, Choi SY, Ju JH, Park YH. Anti-TNFa treatment for HLAB27-positive ankylosing spondylitis-related uveitis. Am J Ophthalmol. 2016;170:32-40. doi:10.1016/j.ajo.2016.07.016

22. Nunes H, Bouvry D, Soler P, Valeyre D. Sarcoidosis. Orphanet J Rare Dis. 2007:2:46. doi:10.1186/1750-1172-2-46

23. Pasadhika S, Rosenbaum JT. Ocular sarcoidosis. Clin Chest Med. 2015; 36(4):669-683. doi:10.1016/j.ccm.2015.08.009

24. Erckens RJ, Mostard RLM, Wijnen PAHM, Schouten JS, Drent M. Adalimumab successful in sarcoidosis patients with refractory chronic non-infectious uveitis. Graefes Arch Clin Exp Ophthalmol. 2012;250(5): 713-720. doi:10.1007/s00417-011-1844-0

25. Riancho-Zarrabeitia L, Calvo-Río V, Blanco R, et al. Anti-TNF-a therapy in refractory uveitis associated with sarcoidosis: Multicenter study of 17 patients. Semin Arthritis Rheum. 2015;45(3):361-368. doi:10.1016/j.semarthrit.2015.05.010

26. Zeidan MJ, Saadoun D, Garrido M, Klatzmann D, Six A, Cacoub P. Behçet's disease physiopathology: A contemporary review. Auto Immun Highlights. 2016;7(1):4. doi:10.1007/s13317-016-0074-1

27. Gül A. Pathogenesis of Behçet's disease: Autoinflammatory features and beyond. Semin Immunopathol. 2015;37(4):413-418. doi:10.1007/ s00281-015-0502-8
28. Fabiani C, Vitale A, Emmi G, et al. Efficacy and safety of adalimumab in Behçet's disease-related uveitis: A multicenter retrospective observational study. Clin Rheumatol. 2017;36(1):183-189. doi:10.1007/ s10067-016-3480-x

29. Minos E, Barry RJ, Southworth S, et al. Birdshot chorioretinopathy: Current knowledge and new concepts in pathophysiology, diagnosis, monitoring and treatment. Orphanet J Rare Dis. 2016;11(1):61. doi:10.1186/s13023-016-0429-8

30. Cunningham ET, Levinson RD, Denniston AK, Brézin AP, Zierhut M. Birdshot chorioretinopathy. Ocul Immunol Inflamm. 2017;25(5):589-593. doi:10.1080/09273948.2017.1400800

31. Huis Het Veld PI, van Asten F, Kuijpers RWAM, Rothova A, de Jong EK, Hoyng CB. Adalimumab therapy for refractory birdshot chorioretinopathy. Retina. 2019;39(11):2189-2197. doi:10.1097/IAE.000000000 0002281

32. Steeples LR, Spry P, Lee RWJ, Carreño E. Adalimumab in refractory cystoid macular edema associated with birdshot chorioretinopathy. Int Ophthalmol. 2018;38(3):1357-1362. doi:10.1007/s10792-017-0592-5

33. Chu XK, Chan C-C. Sympathetic ophthalmia: To the twenty-first century and beyond. J Ophthalmic Inflamm Infect. 2013;3(1):49. doi:10. 1186/1869-5760-3-49

34. Chang GC, Young LH. Sympathetic ophthalmia. Semin Ophthalmol. 2011;26(4-5):316-320. doi:10.3109/08820538.2011.588658

35. Kim JB, Jeroudi A, Angeles-Han ST, Grossniklaus HE, Yeh S. Adalimumab for pediatric sympathetic ophthalmia. JAMA Ophthalmol. 2014;132(8):1022-1024. doi:10.1001/jamaophthalmol.2014.426

36. Hiyama T, Harada Y, Kiuchi Y. Effective treatment of refractory sympathetic ophthalmia with glaucoma using adalimumab. Am J Ophthalmol Case Rep. 2019;14:1-4. doi:10.1016/j.ajoc.2019.01.009 